\title{
Electronic Distractions of the Respiratory Therapist and Their Impact on Patient Safety
}

\author{
Peter J Papadakos MD FAARC
}

\author{
Introduction \\ Addiction to Electronic Devices and Social Media \\ The iPatient \\ Electronic Etiquette \\ Education on Electronic Distraction \\ Summary
}

\begin{abstract}
Over the last decade, data from the lay press, government agencies, and the business world have identified ever-growing problems with electronic distraction and changes in human relationships in this electronically interconnected planet. As health professionals, we are well aware of the epidemic growth of injuries and deaths related to texting and driving. It should not surprise us that this distracted behavior has affected all levels of health-care providers and has impacted patient care. This advent of "distracted doctoring" was first coined by the Pulitzer Prize-winning correspondent Matt Richtel in a landmark article in the New York Times, "As doctors use more devices, potential for distraction grows." This article was a flashpoint for professional organizations to reflect on this change in behavior and how it will impact patient safety and how we relate to patients. The explosion in technology (both personnel and hospital-based), coupled with a rapid social shift, creates an environment that constantly tempts health-care workers to surf the internet, check social media outlets, or respond to e-mails. Studies and commentaries in the medical literature only support how this is a growing problem in patient safety and may both increase medical errors and affects costs and the way we relate to patients and fellow staff. The Emergency Care Research Institute (ECRI) released its annual list of technology hazards for 2013, and three ring true for United States caregivers: distractions from smartphones and mobile devices, alarm hazards, and patient/data mismatches in electronic medical records and other health IT systems, all being in the top 10. How do we begin to address these new technological threats to our patients? First and foremost, we accept that this problem exists. We begin by educating our students and staff that this electronic explosion affects our behavior through addiction and the environment within our hospital through the use of electronic medical records, alarms, and alerts that may impact vigilance and affect our focus. These educational and policy changes should, at their core, address human-to-technology interfacing and teach electronic etiquette. How we approach patients should always have at its core the ancient adage "know thyself"; in other words, always practice self-examination in our daily interactions with technology. Key words: electronic distraction; patient safety; professionalism; electronic medical records; alarm fatigue. [Respir Care 2014;59(8):1306-1309. (C) 2014 Daedalus Enterprises]
\end{abstract}

Dr Papadakos is affiliated with Critical Care Medicine, University of Rochester, Rochester, New York.

Dr Papadakos presented a version of this paper as the 29th Philip Kittredge Memorial Lecture at the 59th AARC Congress, held November 16-19, 2013, in Anaheim, California.
The author has disclosed no conflicts of interest.

Correspondence: Peter J Papadakos MD FAARC, Critical Care Medicine, University of Rochester, 601 Elmwood Avenue, Rochester, NY 14642. E-mail: peter_papadakos@urmc.rochester.edu.

DOI: $10.4187 /$ respcare. 03483 


\section{Introduction}

Over the last decade, there has been an explosion in our society of the use of personal electronic devices (PEDs), smartphones, tablets, and minicomputers. These devices have changed the way we communicate. There has been a shift from verbal communication to device-based interaction. Facebook, Twitter, e-mail, and iChat (to just name a few) have become an obsession for a vast segment of society. It has been reported that, on average, social media

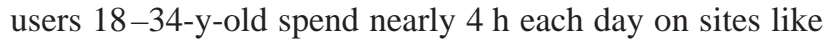
Facebook, Twitter, and LinkedIn, not including e-mail. ${ }^{1}$ This widespread use may affect health professionals, impact health-care delivery, and change the focus from the patient to technology.

In 2011, Matt Richtel reported on a commentary published in Anesthesiology News, ${ }^{2}$ and the term "distracted doctoring" was coined in the front page of the New York Times. ${ }^{3}$ This high impact report started a firestorm of media coverage looking at this issue throughout health-care delivery systems. Many health-care organizations began to address this issue and how it first affects patient safety and, more importantly, how to develop guidelines and protocols to educate staff on how to modulate PED use in professional practice. The respiratory therapy community (in both the United States and Canada) has in many ways been the leader in patient safety and was one of the first professional organizations to address the topic of electronic distraction and electronic patient etiquette.

\section{Addiction to Electronic Devices and Social Media}

At the core of the problem of distracted doctoring over the last 10 years is society's embrace of PEDs and social media. This decade has seen the most rapid embrace of a technology that has effected a complete change in human communication. The staggering explosion in applications (apps) to over 1 million available through Apple alone illustrates how dependent humans have become on their PEDs. ${ }^{4}$

This massive exposure and acceptance of PEDs and connectivity to the internet and social media have affected society as a whole. Taking a few moments and looking around any public place reinforces the fact that the vast majority of the public is walking about holding a PED in hand. Other than the massive increase in accidents due to texting, over 1.3 million crashes, as per the National Safety Council, have been attributed to texting and cell telephone use. Modern man views technology as a magical problemsolver, especially computer-based technology. Thus, because of the countless benefits advanced technology has already bestowed upon us, we rarely have considered its potential downside or its unintended consequences. This infatuation with hand-held technology may be the core of
Table 1. University of Rochester Modified CAGE Questionnaire

(1) Have you ever felt you needed to cut down on the use of your PED?

(2) Have people annoyed you by criticizing your use of your PED?

(3) Have you felt guilty about your overuse of your PED at work?

(4) Do you reach for your PED first thing in the morning?

$\mathrm{PED}=$ personal electronic device, including smartphone, tablet, and minicomputer

what is beginning to be identified as an addictive behavior that not only affects the general public, but may also be at the root of distracted health-care workers.

In developing educational programs to educate the public and health-care providers, we use the step of selfawareness to electronic addiction. ${ }^{5}$ One of the tools used to point out self-addiction is the University of Rochester's modified CAGE questionnaire (Table 1). The CAGE tool is a highly validated survey of alcohol addiction that has been modified to replace drinks with PEDs, is a springboard to starting a dialogue, and can lead to self-correction behavior in the professional environment.6,7

This addiction component has been published in the behavior patterns of 2 distinct groups of health professionals: cardiac perfusionists and pediatric residents. Both groups knew that their use of PEDs took away from patient care but could not stop themselves. ${ }^{8,9}$ This behavior has been volunteered to me by all levels of health-care workers, including therapists at local and national meetings. The entire spectrum of respiratory therapy practice is at risk of this behavior, from the student checking Facebook to the experienced manager juggling hundreds of e-mails dealing with administration of a department. We must not deny that our profession is exempt.

\section{The iPatient}

With the advent of electronic medical records, a new major problem has developed in health care: the so-called iPatient. The health-care practitioner no longer develops a personal relationship with the patient, but has a fixation for the patient's electronic record and data. This mandated use of electronic records may lead to individuals not spending time with the patients and listening to their complaints, the quality of their breathing, and the subtle symptoms of respiratory disease. They are fixed on problem lists, care plans, and other mandated data collection tools.

In this texting and data-filled world, where the majority of interactions are with computers and PEDs, we may risk losing the skill, or even the desire, to communicate verbally, face-to-face. As we look around in the modern work place, this ever-rising world of electronic communication is validated by the hundreds of texts and e-mails we re- 
ceive. Individuals may not ever be aware that they are losing vital listening skills that could otherwise enable us to hear what the patient is really saying. We also will lose the close observational skills of the therapist, the patterns of breathing, and the interplay of the patient and the mechanical ventilator. This interplay gives the therapist the ability to titrate ever-increasing ventilator technology to better care for patients. Therapists should never stop providing hands-on care and observing patients.

Not only is the modern therapist interacting with electronic medical records, his world is one in which he is surrounded by ever-more complex technology with everincreasing alarms and soft stops. The therapist must develop new skills to filter these multiple alarms to focus only on key alarm cues from specific patients and prioritize them to care for ever-more complex patients. If these skills are not taught, the individual health-care practitioner may fall into a vortex of alarm fatigue.

\section{Electronic Etiquette}

I believe that professional and staff education is at the core of preventing electronic distraction, interaction with electronic medical records, and alarm fatigue. Professional schools and hospitals need to place electronic etiquette at the forefront of patient safety education. The lay press is filled with reports of PED distraction in health professionals that has led to bad outcomes, including the death of a patient under anesthesia in Dallas reported widely by the media. The New York Times article ${ }^{3}$ that first introduced distracted doctoring had a vast number of online comments that spoke to the fact that "my health-care worker is not paying attention to me but is looking at a computer screen." This perception needs to be addressed, or it will lead to a breakdown of the sacred relationship between patient and healer.

We mostly teach behavior modification to our students and staff that certain behavior patterns outside the healthcare environment with their addictive qualities play no role in health care. Staff members need to realize that texting, which is information-poor and not Health Insurance Portability and Accountability Act (HIPAA)-safe, is not as effective as face-to-face discussion with a patient or verbal communication.

Staff members need to involve patients in this electronic etiquette. When entering a patient care setting, the practitioner should introduce the patient and family to the technology. I call this the third-person-in-the-room concept, as with a student therapist. For example, I say, "Mrs Jones, we are going to use the computer here to enter all the ventilator settings and blood gases of your husband so that the care team has ready access to his data and can develop a care plan." Thus, the patient's family now fully under- stands that the computer is part of the treatment and not a distraction.

The user should always face the patient and the family when entering information so that the patient knows that the device is being used for medical care and not to surf the internet. This will aid in developing a bond of trust. I cannot say enough about this bond of trust and how it will aid in the healing process. New technology also has many advantages and, if properly integrated, can lead to enhanced patient education and care. Hospitals should develop communication tools that allow face-to-face communication with patients and families through HIPAAprotected networks and interactive tools. Family dynamics in such face-to-face relationships are key, especially in patients who may need end-of-life planning. This warm interaction based on trust, personal awareness and interaction also decreases medical-related legal risks.

Another important factor for the health professional to understand is that patients and their families feel uncomfortable seeing health professionals on their PEDs. This discomfort was a major point in the many commentaries on the New York Times article ${ }^{3}$ and in many discussion groups I have had with patient families. Many of them use their own PEDs to entertain themselves at work and as distractions from work tasks and may believe that the healthcare team is doing the same. Human nature makes them project their behavior patterns onto the health-care team. Remember, they want their health professionals to focus on them and their care. Thus, I recommend that, when in professional dress, health professionals should not be on devices while in elevators and other public places, but only in private during breaks.

Ring tones and alerts on PEDs are also a major aspect of the breakdown of patient relations. Many of us use personalized ringtones that may be very disruptive in a patient care environment, for example, a health professional whose PED rings with loud marimba music while explaining a mode of ventilation to the family of a complex patient with ARDS. This may affect the therapist's family relationship for the worse in that it may be perceived as rude. A good idea would be to place all devices on silent and to inform family and friends to call or text during work hours for emergencies only. Another idea is to have a separate PED for work only, so all calls will be about patients, thus removing the daily distractions of personal texts and alerts. We do not need to be aware of markdown sales at department stores, etc, while caring for patients.

\section{Education on Electronic Distraction}

The most important way to deal with this explosion of electronic distractions, iPatients, and human-to-device interactions is education. We must push such education to start early in the career of therapists and other health pro- 
fessionals. Specific education should start with the selfawareness of addiction that I discussed earlier and cover the skill of electronic etiquette. ${ }^{10}$ Professional schools need to include this training in the safety curriculum with open discussions on how distracted behavior may affect patient outcomes and lead to medical-related legal problems. This education should be included in hospital and departmental orientations of all health-care workers. Several major medical centers have followed the University of Rochester example and have developed guidelines such as the Code of e-Conduct of the University of Rochester Medical Center. This Code of e-Conduct clearly points out how to minimize distraction and how to integrate computer technology with patient care. Roles are defined in the interaction of technology during patient rounds, usually freeing the most senior member of the team from computers for interaction with patients and families. We have found such guidelines helpful, and I encourage their development in departments and hospitals. Through such education, I believe we can decrease electronic distraction and change how we interact with technology to better care for our patients. Therapists need to lead by example since much of our job is technologically rich.

I hope that these thoughts lead to both self-improvement and motivating educators, both professionally and personally, to deal with this overarching fixation with PEDs and social media. I suggest open discussions with colleagues and family members to better modulate technology in our daily lives.

\section{Summary}

As human communication has changed from verbal faceto-face communication to a world dominated by texting, tweets, e-mails, and social media, health professionals must be educated to focus on patient care. Distracted doctoring has become a major patient health concern, and respiratory care practitioners must lead the way in developing programs to guarantee professionalism when interacting with technology. The preservation of human observational skills during patient interaction must be paramount. Programs to eliminate electronic distraction and poor human communication skills must be included in respiratory care curriculum and in all departmental orientation. Through the selfawareness that comes with educational programs, respiratory therapists will become leaders in effecting good practice and become examples for other health professionals.

\section{REFERENCES}

1. Duggan M, Smith, A. Social Media Update 2013. Pew Research Center: December 30, 2013. http://www.pewinternet.org/2013/12/30/ social-media-update-2013. Accessed June 4, 2014.

2. Papadakos PJ. Electronic distraction: an unmeasured variable in modern medicine. Anesthesiology News 2011;37(11):8.

3. Richtel M. As doctors use more devices, potential for distraction grows. New York Times: December 15, 2011. http://www.nytimes. com/2011/12/15/health/as-doctors-use-more-devices-potential-fordistraction-grows.html?pagewanted $=\mathrm{all} \& \_\mathrm{r}=0$. Accessed June 4, 2014.

4. Jones C. Apple's app store about to hit 1 million apps. Forbes: December 11, 2013. http://www.forbes.com/sites/chuckjones/2013/ 12/11/apples-app-store-about-to-hit-1-million-apps. Accessed May 19, 2014.

5. Take a stand. Association of periOperative Nurses: September 18, 2013. http://www.aorn.org/News.aspx?id=10737418310. Accessed May 19, 2014

6. Papadakos PJ. The rise of electronic distraction in health care is addiction to devices contributing. J Anesthesiology Clin Res 2013; 4:e112.

7. Ewing JA. Detecting alcoholism. The CAGE questionnaire. JAMA 1984;252(14):1905-1907.

8. Smith T, Darling E, Searles B. 2010 Survey on cell phone use while performing cardiopulmonary bypass. Perfusion 2011;26(5):375-380.

9. Katz-Sidlow RJ, Ludwig A, Miller S, Sidlow R. Smartphone use during inpatient attending rounds: prevalence, patterns and potential for distraction. J Hosp Med 2012;7(8):595-599.

10. Papadakos PJ. Training health professionals to deal with an explosion of electronic distraction. Neurocrit Care 2013;18(1):115-117. 